\title{
The Role of the Gray Wolf in Rabies Transmission in Iran and Preliminary Assessment of an Oral Rabies Vaccine in this Animal
}

\author{
Alireza Gholami ${ }^{*}$, Siamak Massoudi², Majid Kharazian Moghaddam², Mahmoud Ghazi Marashi², Mahyar \\ Marashi ${ }^{2}$, Rouzbeh Bahsar ${ }^{1}$, Ahmad Fayaz ${ }^{1}$, Maryam Fazeli ${ }^{1}$, Firouzeh Farahtaj ${ }^{1}$, Nader Howaizi ${ }^{1}$, \\ Mohammad-Reza Shirzadi ${ }^{3}$
}

${ }^{1}$ National Reference Center for Rabies, Pasteur Institute of Iran, Tehran, Iran; ${ }^{2}$ Department of Environment, Wildlife Diseases Group, Wildlife Bureau, Tehran, Iran; ${ }^{3}$ Department of Zoonoses, Center for Communicable Disease Control, Ministry of Health and Medical Education, Tehran, Iran

Received Feb 20, 2018; Accepted Feb 24, 2018

\begin{abstract}
Introduction: In Iran, 95\% of animal bites are attributed to domestic dogs and cats, while one-third of rabies victims are from wildlife. The wolf is one of the primary vectors of wildlife in the country. This study was aimed to assess the impact of wildlife on rabies transmission and to evaluate the efficacy of an oral rabies vaccine (ORV) in gray wolf (Canis lupus pallipes). Methods: We studied the incidence of wolf attacks by analyzing a 10-year period of data (2000 to 2009) available in the Ministry of Health and Medical Education, the Veterinary Organization and Pasteur Institute of Iran. In the next step, five captive gray wolves were fed by Raboral V-RG ${ }^{\mathbb{B}}$ vaccine baits $\left(\right.$ Merial ${ }^{\mathbb{R}}$, part of Boehringer Ingelheim, Germany) and monitored for rabies neutralizing antibodies over 20 months by rapid fluorescent focus inhibition test (RFFIT). Results: Our analysis showed that $55 \%$ of animal bites occurred in rural areas and the remaining in urban areas. The most rabid wolves were from Fars province in southern Iran. The vaccine baits were palatable for the hungry wolves. Seroconversion with antibodies titers $\geq 0.5 \mathrm{IU} / \mathrm{ml}$ occurred after 12 weeks and maintained for 78 weeks indicating potential protection against rabies after receiving a single ORV bait. Conclusion: Our results suggested that a single dose of vaccination with $\mathrm{V}-\mathrm{RG}^{\mathbb{R}}$, though initially designed for foxes and raccoons, can protect the gray wolves against rabies as well. J Med Microbiol Infect Dis, 2017, 5 (3-4): 56-61. DOI: 10.29252/JoMMID.5.3.4.56
\end{abstract}

Keywords: Wolf, Rabies, Iran, Oral rabies vaccination, Anti-rabies antibodies.

\section{INTRODUCTION}

Rabies is an acute progressive viral encephalitis and one of the most critical zoonotic diseases worldwide with the fatality rate of almost $100 \%$ [1]. The disease is commonly transmitted through the bite of rabid animals. The etiological agent of the disease is an enveloped singlestranded negative-sense RNA virus belonging to the Rhabdoviridae family and the genus Lyssavirus (Order Mononegavirales). Members of this family occur on all continents except Antarctica [2]. Carnivores are the main reservoirs of the virus in nature and dogs play a significant role in the transmission of rabies to humans in most developing countries. Whereas, wild animals are mainly responsible for the dissemination of rabies in developed countries [3]. Some of these countries have controlled sylvatic cycle of rabies by oral vaccination. Transmission of rabies from wildlife to domestic animals and humans remains a severe public health concern in many countries [4]. In addition to human deaths, rabies is responsible for substantial cattle losses and pose a threat to rare carnivore species $[5,6]$. In northern provinces of Iran, the majority of rabies occurs in livestock kept in barns [7, 8]. Rabies, as an endemic disease, circulates among both wild and domestic animals of Iran. Annually, the national reference center for rabies, Pasteur Institute of Iran, confirms several hundred rabid animals. In addition to the dogs, wild carnivores such as foxes, jackals, and wolves are vectors of rabies in wildlife. Until now, 62 rabies foci have been identified in Iran's wildlife with the disease spillovers to domestic animals in these areas [9, 10]. Previous studies showed that dogs and foxes in the north and wolves in the west and northwest of Iran were the most important vectors of the disease. Dogs were the most frequent potential source of exposure to rabies (62\%) during 1993-1994 and 2008-2009. In Khorasan and Kerman provinces in the east and south of Iran, wild rabies was more critical in comparison to other regions [11, 12]. In Ilam province, western Iran, dogs were responsible for the majority of animals bites (89.2\%) during 1999-2009, followed by cats $(5 \%)$, wolves $(0.7 \%)$, jackals $(0.6 \%)$, foxes $(0.3 \%)$, and other animals (4.2\%) [13]. In Tehran, most of the animal bites were inflicted by dogs $(65.9 \%)$, followed by cats (25.44\%), ferrets (3.98\%), monkies (1.52\%), hamsters (1.41\%), and other animals (1.84\%) [14].

Vaccination of wild animals and dogs can prevent the circulation of the rabies virus in the wildlife and its

*Correspondence: Alireza Gholami

National Reference Center for Rabies, Pasteur Institute of Iran, No. 69, Pasteur Ave, Tehran, Iran, 1316943551

Email: a.gholami@pasteur.ac.ir

Tel: $+98(21) 64112227 \quad$ Fax: $+98(21) 64112813$ 
transmission to domestic animals and humans as well. Annually, about 300-400 thousands owned dogs receive a vaccination by veterinary clinics in Iran. However, this approach does not cover stray dogs. The leftovers and garbage attract stray dogs and wild animals resulting in an increased risk of human exposure to animal bites. This condition also increases the possibility of crossbreeding of dogs and wolves. More than 160,000 animal bites by various animals are reported annually, of which $10.8 \%$ are attributed to wolves (CDC, Ministry of Health and Medical Education (MOHME) of Iran). In some developing countries, preventive vaccination against rabies is recommended for people and especially for children who live in endemic areas. In most endemic regions of the world, $99 \%$ of rabies transmissions to humans are due to the dog bites. Hence, mass vaccination of dogs is considered as the most efficient way to control human rabies [15]. In Iran, dogs and cats are responsible for almost 95\% of the bites, whereas wild animals are responsible for more than onethird of human rabies deaths.

The sylvatic cycle of rabies preserves the virus among wildlife and maintains the likelihood of rabies reemergence [16]. Elimination of rabies in wildlife and prevention of the disease transmission from wildlife to domestic and companion animals in endemic areas can be achieved by mass vaccination campaigns [17]. In developed countries, attempts are directed to control rabies in wildlife, and oral rabies vaccination (ORV) has received considerable attention as a promising and socially acceptable approach. The oral bait vaccines can be distributed over extensive areas in a short period. Until now, several strains of rabies vaccine baits have been developed and applied to the wildlife worldwide [18, 19], and the WHO has approved some oral vaccine baits for rabies control in wildlife [20]. For example, according to the EU requirements, the SAG2 baits were efficient for vaccination of the red fox and raccoon dog, and Raboral V-RG ${ }^{\circledR}$ has been widely used to control rabies in foxes and raccoons [21, 22]. This recombinant anti-rabies vaccine, due to using a live virus, is only recommended for wild animals. The recombinant vaccinia virus is stable at elevated temperatures and can be delivered orally. Different reports showed the elimination of sylvatic rabies by using Raboral V-RG ${ }^{\circledR}$ in United States, Canada, and several other countries [23].

History of the wolf associated rabies in Iran demonstrates the aggressive behavior and excessive physical power of this animal in inflicting deep and numerous injuries to the victim's body. Hence, the rabid wolf has long been considered as one of the most dangerous carriers of the disease in wildlife of Iran [24]. The current study has analyzed the role of wild animals in the transmission of rabies in Iran and the ability of Raboral V$\mathrm{RG}^{\circledR}$ as an ORV in the production of neutralizing antibodies against rabies virus in the captive gray wolves (Canis lupus pallipes).

\section{MATERIAL AND METHODS}

Vaccines. We used Raboral V-RG ${ }^{\circledR}$ vaccine baits for oral vaccination. Each bait block (size: $\sim 1$ 1/4" X 1 1/4") contained $2 \mathrm{ml}$ of recombinant vaccinia virus $\left(10^{7.7}\right.$ plaque forming units per $\mathrm{ml}$ ) encoding the rabies virus surface glycoprotein, coated by fishmeal. For comparison, Rabisin vaccine was used for vaccination through parenteral route. The commercial company of Merial ${ }^{\circledR}$ manufactures both vaccines.

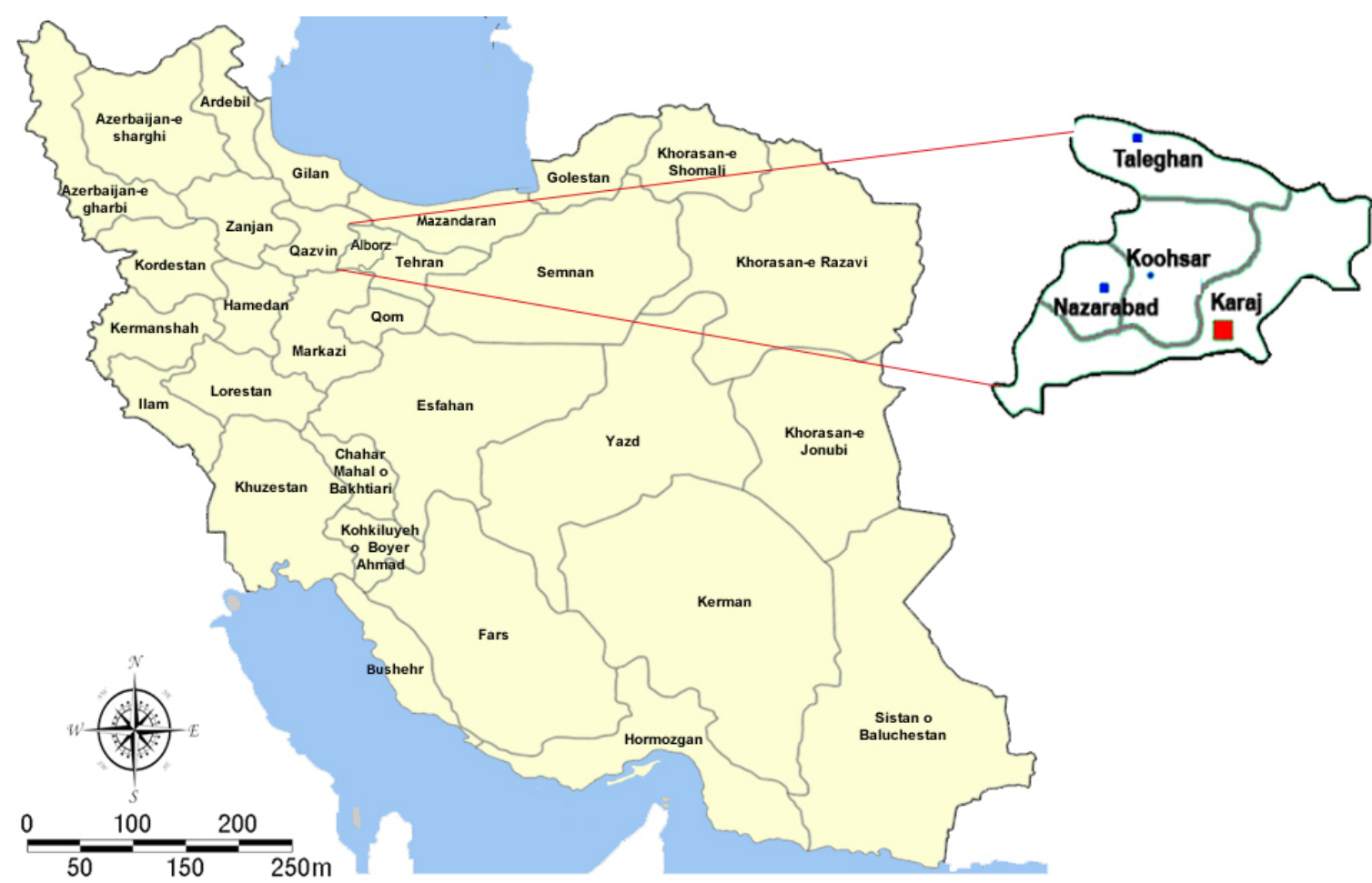

Fig. 1. Location of Koohsar town (shown by the red square) in Alborz province of Iran, where the wolves were kept, and the vaccination study was performed 
Animals. Five adult healthy captive gray wolves (Canis lupus pallipes) including two males and three females with an average weight of 36 and $26 \mathrm{~kg}$, respectively were used in this study. A group of four wolves was fed by vaccine baits, and a single wolf received vaccination through parenteral route. Production of neutralizing antibodies was monitored for 16 months. This interventional study was performed at wildlife rehabilitation center of Issar recreation and cultural complex, Koohsar county, Karaj, Iran (Fig. 1).

Vaccine Administration. Before feeding with baits, the animals were starved for $24 \mathrm{~h}$, and then each animal in the test group was allowed to feed on one bait. The animals' reaction to the baits was filmed.

Measuring of anti-rabies antibodies. We collected blood from wolves on days $0,14,90,360$ and 480 postvaccination by a veterinarian surgeon. To minimize injuries, the animals were anesthetized with a blowpipe gun containing $5 \mathrm{mg}$ Medetomidine 1\% (Orbene Co., Spain) and $250 \mathrm{mg}$ Ketamine 10\% (Alfasan Co., Netherland). Samples of $5 \mathrm{ml}$ of blood were collected from each wolf using non-heparinized syringes and preserved at $-20^{\circ} \mathrm{C}$ until used. Finally, blood sera were analyzed by rapid fluorescent focus inhibition test (RFFIT) to measure anti-rabies antibodies. Vital signs of animals were closely monitored while taking the blood sampling, and side measures such as administration of antibiotics and other examinations were performed for the animals' well being. The animals were reversed with $200 \mathrm{mcg} / \mathrm{kg}$ in $0.4 \mathrm{ml} / 10 \mathrm{~kg}$ of Atipamezole hydrochloride. A microchip was implanted subcutaneously in each animal for further examinations.

Statistical analysis of data. Statistical analysis of the data obtained from the Veterinary Organization and Department of Environment of the Islamic Republic of Iran were performed by the SPSS software version 9 (SPSS Inc., Chicago, USA). Statistics were given in the form of mean \pm SD along with the median.

\section{RESULTS}

Geographic occurrence of animal bites. According to the statistical analysis, 55\% of animal bites occur in rural areas and the rest in urban areas. The most abundant rabid wolves' population were from Fars province followed by Hamadan, Zanjan, East Azerbaijan, West Azerbaijan and Kurdistan provinces. There was no report of the rabid wolf from South Khorasan, Sistan and Baluchistan, Qom and Bushehr provinces. Prevalences of rabid animals in different provinces are shown in Figure 2.

The prevalence of rabies among the wolves' populations showed an incremental trend during 2000-2009 with the highest incidence in 2002 (Fig. 3). The data showed that the prevalence of rabies in wolves and foxes was higher than other wild animals (0.2\%) (Fig. 4). Besides, wolves were the second most important wild animal causing human rabies (9\%) (Fig. 5).

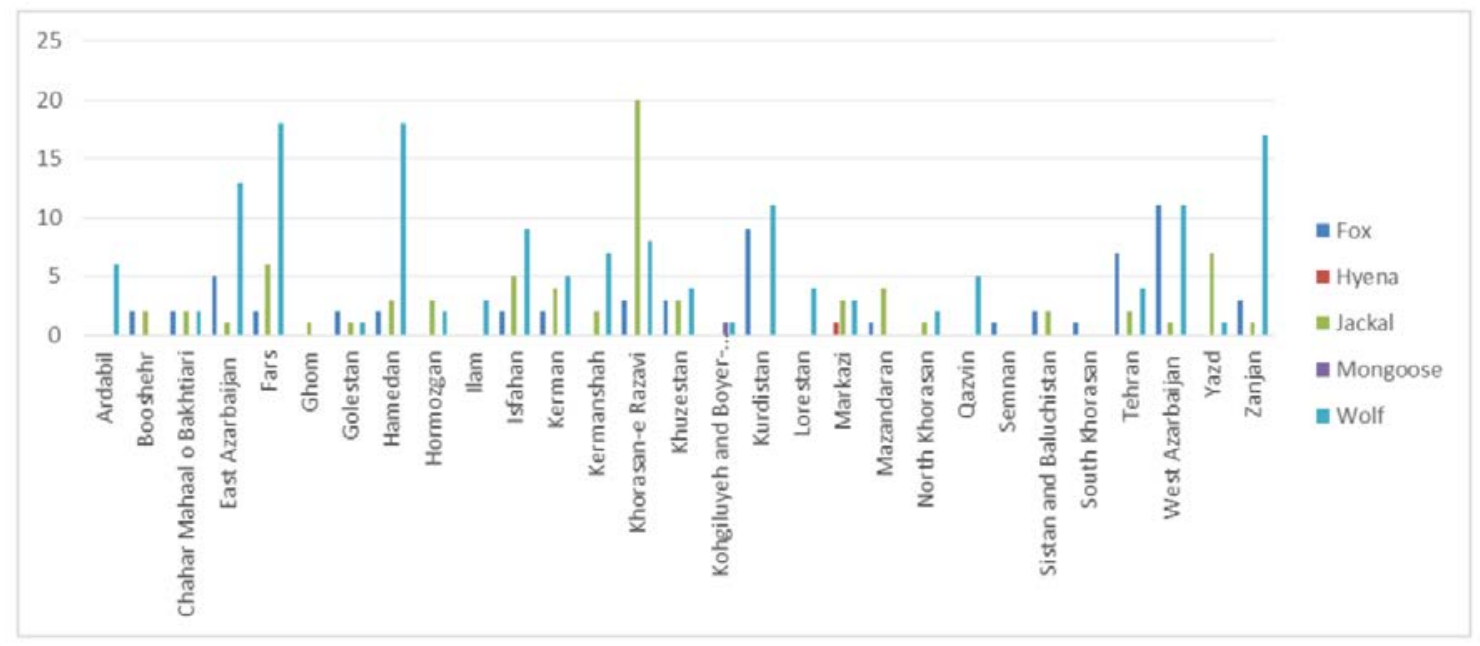

Fig. 2. Prevalence of rabies among wild animals in different provinces of Iran in a 10-year period (2000-2009)



Fig. 3. Prevalence of rabies in wild animals during 2000-2009 in Iran 


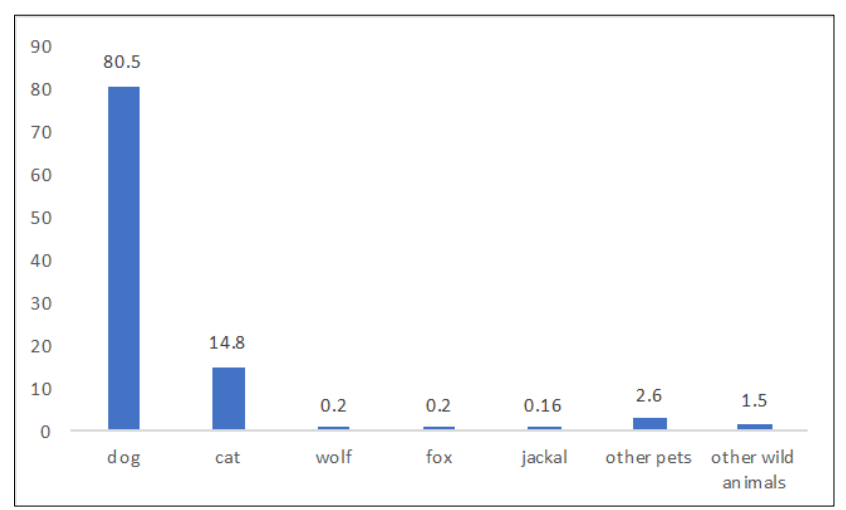

Fig. 4. Prevalence (percentage) of rabies in different animals from 2000 to 2009 in Iran

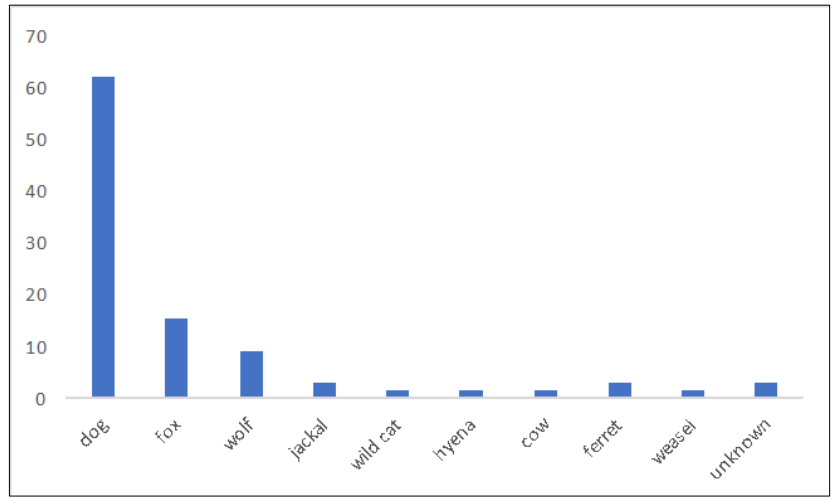

Fig. 5. Percentage of human rabies caused by various species of animals in a 10-year period (2000-2009) in Iran
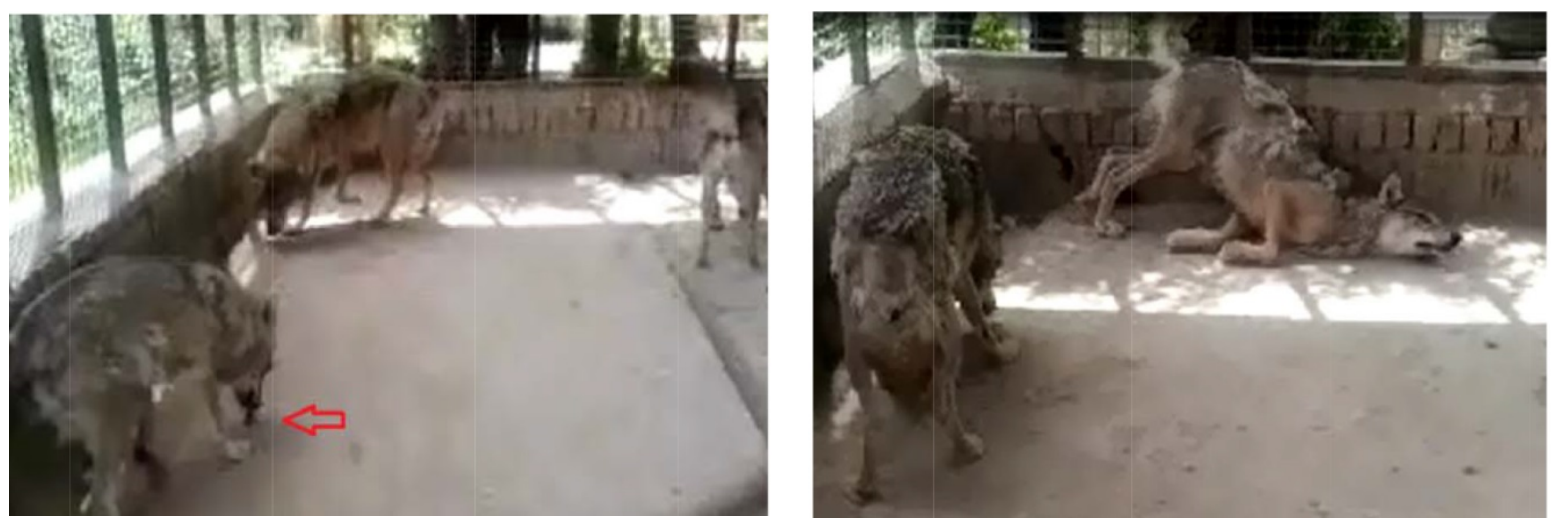

Fig. 6. Wolves reaction to vaccine baits, (left) attraction of the hungry wolves to the baits (shown by a red arrow), (right) a wolf rolling on the bait trying to break it

Wolves Reaction to the bait. The wolves showed particular reactions to the vaccine baits not observed in raccoon and foxes [25, 26, 27]. Some wolves tried to break the bait by rolling over it, and some broke it by teeth, throwing away the paraffin content and vaccine within it, eating only its coating meal (Fig. 6). These animals were anesthetized, and the vaccine capsule inside the bait was instilled onto the tongue and oral cavity by hand. Muscle relaxation in all animals was regular, and palpebral reflexes were present after anesthesia.

RFFIT tests. The test was carried out in the accredited laboratory of the WHO-collaborating center for Reference and Research on Rabies, Pasteur Institute of Iran, according to a WHO protocol $[28,29]$. At the day 90 post-vaccination, the titers were over or equal to $3.3 \mathrm{IU} / \mathrm{ml}$ for all vaccinated wolves. The titers remained over $0.5 \mathrm{IU} / \mathrm{ml}$ over 78 weeks in all wolves indicating sufficient protection against potential rabies infection [30].

\section{DISCUSSION}

According to previous reports [24], and the present study, foxes (Vulpes vulpes), wolves (Canis lupus pallipes) and jackals (Canis aureus), due to their susceptibility to rabies virus and geographical distribution, are among the most important wild carnivores responsible for rabies transmission in Iran. Our analysis showed that $\approx 55 \%$ of animal bites occurred in rural areas with the rabid wolves as the second most important cause of human rabies. These data also emphasized on adopting a strategy for controlling rabies in wild animals especially wolves. In rabid endemic countries, several strategies including the oral rabies vaccination and capture-neuter-vaccinate-release programs, point zoonoses controls, and population management of vector animals have been implemented to control rabies [29]. The concept of oral rabies vaccination efficacy in wild carnivores has been proved since three decades ago [31]. However, in developing countries implementation of rabies control programs using oral vaccines has been a challenge (REF). The V-RG ${ }^{\circledR}$ recombinant vaccine showed a great promise as an oral vaccine for wildlife during the 1980s when the experiments were conducted on captive raccoons and foxes [32]. Unlike similar studies on ORVs [19], in our study, seroconversion was not observed until two weeks. However, the level of anti-rabies antibodies after seroconversion remained over $0.5 \mathrm{IU} / \mathrm{ml}$ in all vaccinated wolves suggesting the efficacy of Raboral V-RG ${ }^{\circledR}$ as an effective vaccine for wolves.

Despite significant scientific progress, more than 60,000 deaths from rabies are still reported worldwide annually, and millions of others require post-exposure treatment [33]. Development of the disease can be prevented in human by 
appropriate wound care and one of the standard postexposure prophylaxis (PEP) regimens [34]. However, PEP is relatively expensive and not always available. PEP alone lacks long-term benefits because it can neither stop the virus in the animal hosts nor its transmission to humans [35].

The implementation of rabies control programs using ORV has been a challenge for veterinary authorities, particularly in densely populated areas in Central Europe [36]. Most new modified-live rabies virus oral vaccines originate from the attenuated Evelyn-Rokitnicki-Abelseth (ERA) virus strain, which was derived from the original Street-Alabama-Dufferin (SAD) rabies virus strain [37]. The first oral rabies vaccine (ORV) strain was SAG2 (Street-Alabama-Dufferin [SAD]-avirulent-gif) that was mutated from the SAD Bern strain by utilizing antiglycoprotein monoclonal antibodies. No biological safety issues have been reported with the SAG2 strain vaccination, and all vaccinated dogs and raccoons showed protection when challenged with the virulent RABV [20, 38]. Another ORV strain, a recombinant deleted E1/E3 Adenovirus strain expressing rabies glycoprotein, could induce production of neutralizing antibodies in dogs, skunks, and raccoons [38]. Other ORVs, like a canarypox-rabies glycoprotein recombinant vaccine, and a recombinant pseudorabies that expresses RABV glycoprotein, showed to be safe and effective for animals [39, 40].

Fighting rabies requires knowledge of the disease cycle between human, wildlife and domestic animals. Of course, the disease control in wildlife is different from preventive measures for domestic animals. In urban areas, population control programs aimed at culling of stray animals were unsuccessful due to economic, ecological and ethical reasons [3]. From observations on the relationship between vaccination coverage and rabies incidence in dogs, it was concluded that rabies outbreaks could be prevented if at least $70 \%$ of the dogs were immunized [17]. The success of wildlife rabies control program depends upon timely and accurate rabies surveillance data, so that response to rabies outbreaks could be immediate with no time lags. Raboral $\mathrm{V}-\mathrm{RG}^{\circledR}$ was approved for immunization of raccoons and coyotes, the two most significant rabies carriers in wildlife of North America [41]. Unlike raccoons and coyotes, in the present study, production of antibodies in captive wolves did not occur until 10-14 days after eating vaccine baits. Further studies on reactions of different wild animals to the vaccine baits and the vaccine uptake by them [42] would help us to develop appropriate baits for various carnivores living in different geographical areas Iran. Also, largerscale studies on non-target animals are required to ensure its efficacy in control of wildlife rabies before widespread implementation of ORVs in Iran.

\section{ACKNOWLEDGEMENT}

This work was supported by the grant (No. 3.139, dated December 24, 2011) provided by Department of Environment, Wildlife Diseases Group, Wildlife Bureau, Tehran, Iran and Pasteur Institute of Iran.

\section{CONFLICT OF INTEREST}

The authors declare that there are no conflicts of interest associated with this manuscript.

\section{REFERENCES}

1. Sudarshan M, Madhusudana S, Mahendra B, Rao N, Narayana DA, Rahman SA, et al. Assessing the burden of human rabies in India: results of a national multi-center epidemiological survey. Int J Infect Dis. 2007; 11 (1): 29-35.

2. Woldehiwet Z. Rabies: recent developments. Res Vet Sci. 2002; 73 (1):17-25.

3. Rupprecht C, Hanlon C, Slate D. Oral vaccination of wildlife against rabies: opportunities and challenges in prevention and control. Dev Biol. 2003; 119: 173-84.

4. Rupprecht CE, Hanlon CA, Hemachudha T. Rabies reexamined. Lancet Infect Dis. 2002; 2 (6): 327-43.

5. Okell C, Pinchbeck G, Stringer A, Tefera G, Christley R. A community-based participatory study investigating the epidemiology and effects of rabies to livestock owners in rural Ethiopia. Prev Vet Med. 2013; 108 (1): 1-9.

6. Bahonar A, Rashidi H, Simani S, Fayaz A, Haghdoost A, Rezaei-nassab M, et al. Relative frequency of animal rabies and factors affecting it in Kerman province, 1993-2003. J Sch Public Health Inst Public Health Res. 2007; 5 (1): 69-76.

7. Shirzadi M, Pourmozafari J, Shamsipour M. Status of Animal Bite and Rabies Cases in Northeast Provinces of Iran during 2009-2010. J Zoonoses. 2014; 1 (1).

8. Esfandiari B, Youssefi MR, Fayaz A. Serodiagnosis evaluation of rabies and animal bites in North of Iran, 2010. J Gen Mol Virol. 2011; 3 (5): 71-3.

9. Shariati A. Epidemiology of rabies in Khorasan state during 1994-2003. Prev Vet Dis. 2004: 90.

10. Simani S. Rabies situation in Iran. J Veter Faculty. 2003; 58 (3): $275-8$.

11. Rezaeinasab M, Rad I, Bahonar A, Rashidi H, Fayaz A, Simani S, et al. The prevalence of rabies and animal bites during 1994 to 2003 in Kerman province, southeast of Iran. Iran J Vet Res. 2007; 8 (4): 343-50.

12. Wilde H, Khawplod $\mathrm{P}$, Khamoltham $\mathrm{T}$, Hemachudha $\mathrm{T}$, Tepsumethanon V, Lumlerdacha B, et al. Rabies control in south and southeast Asia. Vaccine. 2005; 23 (17): 2284-9.

13. Rostampour F, Fallahi A. An epidemiologic study of animal bites in Ilam Province, Iran. Arch Iran Med. 2012; 15 (6): 356.

14. Eslamifar A, Ramezani A, Razzaghi-Abyaneh M, Fallahian V, Mashayekhi P, Hazrati M, et al. Animal bites in Tehran, Iran. Arch Iran Med. 2008; 11 (2): 200-2.

15. Cleaveland S, Kaare M, Knobel D, Laurenson MK. Canine vaccination-providing broader benefits for disease control. Vet Microbiol. 2006; 117 (1): 43-50.

16. Thompson R, Kutz SJ, Smith A. Parasite zoonoses and wildlife: emerging issues. Int J Environ Res Public Health. 2009; 6 (2): 678-93.

17. Coleman PG, Dye C. Immunization coverage required to prevent outbreaks of dog rabies. Vaccine. 1996; 14 (3): 185-6. 
18. Brochier B, Blancou J, Thomas I, Languet B, Artois M, Kieny M-P, et al. Use of recombinant vaccinia-rabies glycoprotein virus for oral vaccination of wildlife against rabies: innocuity to several non-target bait consuming species. J Wildl Dis. 1989; 25 (4): 540-7.

19. Zhugunissov K, Bulatov Ye, Taranov D, Yershebulov Z, Koshemetov Zh, Abduraimov Ye, et al. Protective immune response of oral rabies vaccine in stray dogs, corsacs and steppe wolves after a single immunization. Arch Virol. 2017.

20. Cliquet F, Gurbuxani J, Pradhan H, Pattnaik B, Patil S, Regnault A, et al. The safety and efficacy of the oral rabies vaccine SAG2 in Indian stray dogs. Vaccine. 2007; 25 (17): 3409-18.

21. Pastoret P-P, Brochier B. The development and use of a vaccinia-rabies recombinant oral vaccine for the control of wildlife rabies; a link between Jenner and Pasteur. Epidemiol Infect. 1996; 116 (03): 235-40.

22. Pastoret P, Brochier B. Epidemiology and control of fox rabies in Europe. Vaccine. 1999; 17 (13-14): 1750-4.

23. Slate D, Rupprecht CE, Rooney JA, Donovan D, Lein DH, Chipman RB. Status of oral rabies vaccination in wild carnivores in the United States. Virus Res. 2005; 111 (1): 68-76.

24. Gholami A, Farahtaj F, Fayaz A. Rabies in Iran: Past, Present, and Future. J Med Microbiol Infect Dis. 2014; 2 (1) 110.

25. Slate D, Algeo TP, Nelson KM, Chipman RB, Donovan D, Blanton JD, et al. Oral rabies vaccination in North America: opportunities, complexities, and challenges. PLoS Negl Trop Dis. 2009; 3 (12): e549.

26. Rosatte RC, Power MJ, Machines CD, Campbell JB. Trapvaccinate-release and oral vaccination for rabies control in urban skunks, raccoons and foxes. J Wildl Dis. 1992; 28 (4): 562-71.

27. Krämer B, Schildger H, Behrensdorf-Nicol H, Hanschmann $\mathrm{K}$, Duchow K. The rapid fluorescent focus inhibition test is a suitable method for batch potency testing of inactivated rabies vaccines. Biologicals. 2009; 37 (2): 119-26.

28. Feyssaguet M, Dacheux L, Audry L, Compoint A, Morize J, Blanchard I, et al. Multicenter comparative study of a new ELISA, PLATELIA ${ }^{\mathrm{TM}}$ RABIES II, for the detection and titration of anti-rabies glycoprotein antibodies and comparison with the rapid fluorescent focus inhibition test (RFFIT) on human samples from vaccinated and non-vaccinated people. Vaccine. 2007; 25 (12): 2244-51.

29. Used NIC. Tactics and Economics of Wildlife Oral Rabies Vaccination, Canada and the United States.

30. WHO Expert Consultation on Rabies: second report, 2013, World Health Organization; IRIS. [Cited 2018 March 3].
Available from: http://apps.who.int/iris/bitstream/10665/85346/1/9789240690943_eng.pdf.

31. Black JG, Lawson KF. The safety and efficacy of immunizing foxes (Vulpes vulpes) using bait containing attenuated rabies virus vaccine. Can J Comp Med. 1980; 44 (2): 169-76.

32. Rupprecht C, Kieny M-P. Development of a vaccinia-rabies glycoprotein recombinant virus vaccine. Rabies: Springer; 1988. 335-64.

33. Meslin F, Fishbein D, Matter H. Rationale and prospects for rabies elimination in developing countries. Curr Top Microbiol Immunol. 1994; 187: 1.

34. Warrell MJ. Current rabies vaccines and prophylaxis schedules: preventing rabies before and after exposure. Travel Med Infect Dis. 2012; 10 (1): 1-15.

35. Bögel K, Meslin F. Economics of human and canine rabies elimination: guidelines for programme orientation. Bull World Health Organ. 1990;68(3):281.

36. Selhorst T, Müller T, Bätza H. Epidemiological analysis of setbacks in oral vaccination in the final stage of fox rabies elimination in densely populated areas in Germany. Dev Biol. 2005; 125: 127-32.

37. Rupprecht CE, Hamir AN, Johnston DH, Koprowski H. Efficacy of a vaccinia-rabies glycoprotein recombinant virus vaccine in raccoons (Procyon lotor). Rev Infect Dis. 1988; 10 (Supplement 4): S803-S9.

38. Orciari LA, Niezgoda M, Hanlon CA, Shaddock JH, Sanderlin DW, Yager PA, et al. Rapid clearance of SAG-2 rabies virus from dogs after oral vaccination. Vaccine. 2001; 19 (31): 4511-8.

39. Taylor J, Meignier B, Tartaglia J, Languet B, VanderHoeven J, Franchini G, et al. Biological and immunogenic properties of a canarypox-rabies recombinant, ALVAC-RG (vCP65) in nonavian species. Vaccine. 1995; 13 (6): 539-49.

40. Yuan Z, Zhang S, Liu Y, Zhang F, Fooks AR, Li Q, et al. A recombinant pseudorabies virus expressing rabies virus glycoprotein: safety and immunogenicity in dogs. Vaccine. 2008; 26 (10): 1314-21.

41. Cliquet F, Barrat J, Guiot A, Cael N, Boutrand S, Maki J, et al. Efficacy and bait acceptance of vaccinia vectored rabies glycoprotein vaccine in captive foxes (Vulpes vulpes), raccoon dogs (Nyctereutes procyonoides) and dogs (Canis familiaris). Vaccine. 2008; 26 (36): 4627-38.

42. Vos A, Freuling CM, Hundt B, Kaiser C, Nemitz S, Neubert A, Nolden T, Teifke JP, Te Kamp V, Ulrich R, Finke S, Müller $\mathrm{T}$. Oral vaccination of wildlife against rabies: Differences among host species in vaccine uptake efficiency. Vaccine. 2017 13; 35 (32): 3938-44. 